\title{
FEM ASSISTED OBSERVATIONS OF CUTTING FORCES, TEMPERATURE AND CHIP CURLING IN OBLIQUE MACHINING
}

\author{
Salman Pervaiz \\ Department of Mechanical and Industrial Engineering \\ Rochester Institute of Technology - Dubai Campus \\ P.O. Box 341055 Dubai, United Arab Emirates \\ sxpcad@rit.edu
}

\author{
Sathish Kannan \\ Department of Mechanical Engineering \\ American University of Sharjah \\ P.O. Box 26666 Sharjah, United Arab Emirates \\ skannan@aus.edu
}

\begin{abstract}
It is easier to numerically model the machining process assuming the orthogonal cutting conditions, however the realistic industrial machining arrangements are closer to the oblique machining conditions. In oblique machining the cutting edge is located at a certain inclination angle resulting in a third radial force component. The inclination angle in oblique machining arrangement has a controlling influence over the chip curling and resulting plastic deformation is more complex as compared to the orthogonal machining arrangements. The current study has incorporated a threedimensional finite element model to predict the machining of Inconel 718 using SIALON cutting tool materials under oblique machining conditions. The study incorporates different cutting edge-based inclination angles and resulting simulated machining performance has been reported. The machining performance has been evaluated by observing the simulated cutting forces, cutting temperature, and chip curling.
\end{abstract}

Keywords-component; chip curling, oblique machining, FEM

\section{INTRODUCTION}

As compared to the orthogonal machining setup, oblique machining provides more realistic cutting scenario with respect to the practical application in the metal cutting sector. The predictions about the machining performance under oblique machining arrangement is more reliable and closer to the practical application. Machining performance of a cutting process can be predicted using either analytical or numerical approaches. Numerical approaches are easier to deal with complex geometries and boundary conditions but at the expense of higher computational cost and time. When it comes to the oblique machining performance prediction most of the literature is based on the analytical modelling and very limited studies are available using numerical modeling approaches especially for difficult-to-machine materials such as Inconel 718.

Several researchers have focused their research work to utilize numerical modeling techniques for the prediction of different machining performance indicators. Shaw et al. [1] initially introduced the concept about the normal rake angle. The concept was further evolved in the work conducted by researchers such as Stabler [2,3], Brown and Armarego [4], and Russell and Brown [5] etc. Luk [6] investigated the chip flow under oblique machining setup. The study was conducted using analytical solution method considering mechanistic approach. Lin et al. [7] explored the orthogonal plane strain machining theory to predict the cutting forces under oblique machining conditions. The study revealed that cutting forces can be predicted using the combined knowledge of cutting parameters, materials flow stress and thermal behavior. Arsecularatne et al. [8] investigated the tool life under oblique cutting. The study utilized the theory of tool life using cutting temperature and investigated the tool life for oblique conditions. The experimental validation of predicted results was performed using the plain carbon steel with different tool geometries and cutting conditions.

Lazoglu and Islam [9] utilized finite difference based numerical method to explore the cutting temperature related 3D simulations under oblique cutting arrangements. The predicted results were found to be in good agreement with the experimental findings. Komanduri et al. [10] investigated the influence of both normal rake and inclination angles for the oblique machining of single crystal aluminum using 3D molecular dynamics simulations. The study measured cutting force components, cutting force ratio and specific energy. The study revealed that normal rake angle has controlling influence over the cutting force components and specific cutting energy. Feng and Zang [11] studied oblique machining process using a thermo-elastic-plastic coupled finite element (FE) model. The study was conducted using high speed steel (H11) and ANSI1045 steel as cutting tool and workpiece materials respectively. The study monitored cutting forces, stresses and cutting temperatures at the cutting interface and chips. The study revealed that role of inclination angle in decreasing cutting forces is very minor.

Song et al. [12] developed a model to predict the cutting forces using the vector transformation method and basic mechanistic approach towards the friction at tool - chip interface. The study considered that friction at interface is same in the orthogonal cutting as well as with different inclination angles. The simulated results were found to be in good agreement with experimental data. Grzesik and Zak [13] investigated the surface integrity of different iron-based 
materials such as carbon, low-alloyed, stainless steel and spheroidal iron under oblique machining conditions. The study has incorporated carbide and $\mathrm{Al} 2 \mathrm{O} 3-\mathrm{TiC}$ ceramic cutting tools using inclination angle of $55^{\circ}$. The study revealed the potential of oblique machining to replace conventional finish turning and grinding operations as at higher feed rates oblique turning provides higher surface finish. The study also revealed that maximum value of compressive stress was produced closer to the machined surface, and sensitive to the strain hardening exponent of the workpiece material.

Moufki et al. [14] conducted a study to develop a thermomechanical modelling approach for the oblique machining and simulated results were validated experimentally as well. The study utilized an analytical method based on materials strain rate sensitivity, strain hardening and thermal softening. The model provided predictions for the cutting forces and chip flow. The model was developed for the continuous chip conditions. The model provided predictions in close agreement with the experimental readings. Abdellaoui and Bouzid [15] developed a thermo-mechanical methodology to model the oblique machining. The study developed a computational algorithm and controlled the oblique machining parameters using a ratio of tool-chip contact length and chip ratio. The predicted results were found in good agreement with the experimental findings.

Grzesik and Zak [16] in another study performed friction quantification under oblique machining arrangements using CBN chamfered cutting tools. The study developed an analytical-empirical model of friction. The study incorporated the effect of equivalent rake face and associated equivalent rake angle. The study was conducted on spheroidal cast iron. The study revealed that at large negative rake angles, friction was highly influenced by the inclination angles. Llanos et al. [17] conducted a study where machining performance of AISI4140 steel was investigated using uncoated cemented carbide cutting tools. The study utilized finite element (FE) based model using Arbitrary Lagrangian Eulerian (ALE) formulation. The study revealed that accurate chip formation is linked with the proper identification of frictional behavior at the tool - chip interface. Pawade et al. [18] conducted an analytical study to predict the specific shear energy for Inconel 718 under oblique machining conditions. The study incorporated the Inconel 718 material behavior using Johnson-Cook (JC) material model. JC model can incorporate strain hardening, strain rate sensitivity and thermal softening. The study revealed that shear band spacing linearly increases by increasing the feed rate. The specific shear energy model also revealed that it is more sensitive to thermal softening, strain hardening and shearing strain. The predictions were found in very good agreement with the experimental findings.

It is well understood by the metal cutting community that oblique machining arrangements are more practical than the orthogonal cutting arrangements. However, it has been observed that there is very limited finite element based numerical studies available in literature when comes to the oblique machining. It is even more rare to find in literature that such numerical models are developed for difficult-to-cut materials using modern cutting tool materials. The aim of this study is to utilize a finite element (FE) based numerical approach to predict the machining performance of Inconel 718 under oblique machining conditions.

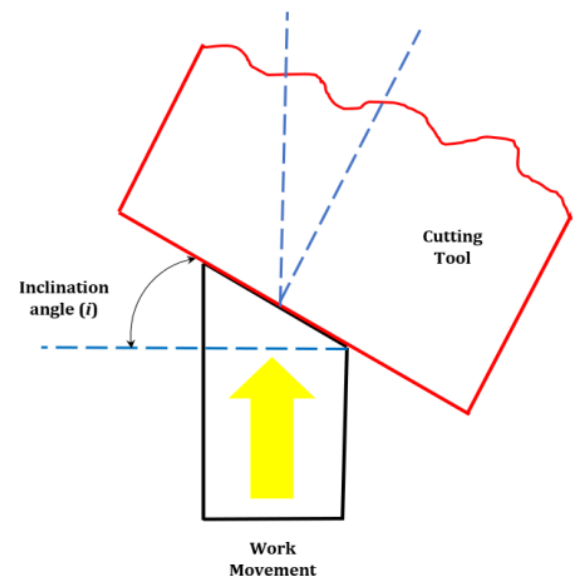

(a)

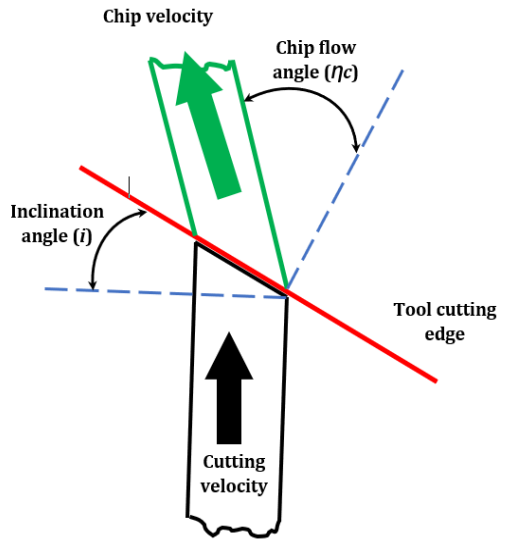

(b)

Figure 1. Schematic illustration of oblique machining (a) Inclination angle (i), and (b) Chip flow angle (Пc) (redrawn from [10], [19])

\section{OBLIQUE MACHINING VIEW}

In literature it is very frequent to find the numerical models for orthogonal cutting (2D) arrangements. Oblique cutting (3D) is different to orthogonal cutting due to the presence of inclination angle $(i)$ as shown in Fig. 1a and 1b.

Inclination angle $(i)$ is the angle that tool cutting edge makes with the normal to the cutting velocity vector. Due to the presence of inclination angle, chip flow is also at an angle other than the right angle as seen in orthogonal cutting case.

\section{FINITE ELEMENT MODELING}

Finite element (FE) model was prepared by using a customized software for machining application known as Thirdwave AdvantEdge [20]. In Thirdwave AdvantEdge software, chip formation is simulated by utilizing dynamic explicit lagrangian formulation. The software can execute coupled thermo-mechanical transient analysis and adaptive remeshing techniques. The software was first presented by Marusich and Ortiz [21]. Thirdwave AdvantEdge has built in

Identify applicable sponsor/s here. (sponsors) 
material library for the cutting tools and workpieces. Fig. 2 represents the schematic illustration from Thirdwave AdvantEdge.

To utilize different engineering materials for different applications, there is a built-in material library in the software package. These material models can mimic the materials behavior by simulating flow stresses, strain rate sensitivity and temperature-based effects (thermal softening). The current study was based on the oblique machining (3D) setup, where cutting tool material was selected to be SIALON and workpiece material was selected to be Inconel 718. By default, AdvantEdge software utilizes power law to model the constitutive behavior of the Inconel 718. The constitutive power law can incorporate the effects related to strain hardening, strain rate sensitivity and thermal softening. The power law is mentioned in Eq. 1 .

$$
\sigma\left(\varepsilon^{P}, \dot{\varepsilon}, T\right)=g\left(\varepsilon^{P}\right) \Gamma(\dot{\varepsilon}) \Theta(T)
$$

In Eq. $1, g\left(\varepsilon^{P}\right), \Gamma(\dot{\varepsilon})$ and $\Theta(T)$ represent strain hardening, strain rate sensitivity and thermal softening respectively, and the functions are further elaborated in equations 2, 3 and 4 [22].

$$
\begin{aligned}
& \left(\varepsilon^{P}\right)=\sigma_{0}\left(1+\frac{\varepsilon^{P}}{\varepsilon_{0}^{P}}\right)^{1 / \mathrm{n}} \\
& \Gamma(\dot{\varepsilon})=\sigma_{0}\left(1+\frac{\dot{\varepsilon}}{\dot{\varepsilon}_{0}}\right)^{1 / \mathrm{m}} \\
& \Theta(T)=C_{0}+C_{1} T^{1}+\cdots C_{5} T^{5}
\end{aligned}
$$

In Eqs. 2, 3 and 4, $\sigma_{0}$ represents initial yield stress, $\varepsilon^{P}$ represents plastic strain, $\varepsilon_{0}^{P}$ represents reference plastic strain, $\dot{\varepsilon}$ represents strain rate, $\dot{\varepsilon}_{0}$ is reference plastic strain rate, $\mathrm{C} 0-\mathrm{C} 5$ represents coefficients for the polynomial fit, $\mathrm{T}$ is temperature, $\mathrm{n}$ represents strain hardening exponent, and $\mathrm{m}$ represents strain rate sensitivity coefficient [20].

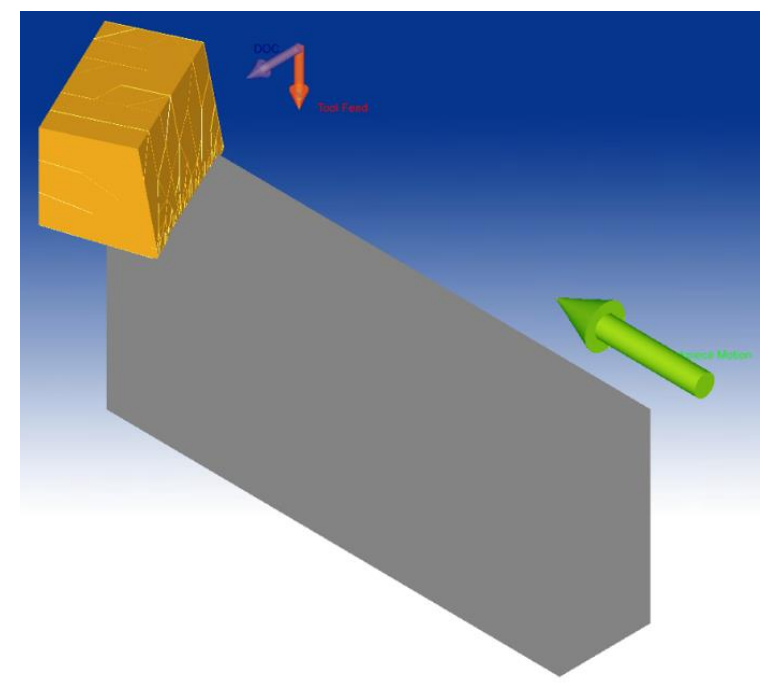

Figure 2. Schematic illustration of oblique machining in Thirdwave AdvantEdge
TABLE 1. PARAMETERS USED FOR NUMERICAL SIMULATIONS.

\begin{tabular}{|lc|}
\hline Simulation Parameters & Values \\
\hline Cutting Tools & SAILON \\
Workpiece Materials & Inconel 718 \\
Normal Rake angle $\left(^{\circ}\right)$ & 10 \\
Cutting Speed $(\mathrm{m} / \mathrm{min})$ & 300 \\
Feed rate $(\mathrm{mm} / \mathrm{rev})$ & 0.15 \\
Depth of Cut $(\mathrm{mm})$ & 1 \\
Length of Cut $(\mathrm{mm})$ & 3 \\
Friction Co-efficient & 0.5 \\
Lead angle $\left({ }^{\circ}\right)$ & 0 \\
Initial Temperature $\left(^{\circ}\right)$ & 20 \\
Relief Angle $\left({ }^{\circ}\right)$ & 5 \\
Inclination Angle $\left(^{\circ}\right)$ & $0,5,10,15,20,25$ \\
Cutting Environment & Dry \\
\hline
\end{tabular}

Like other FE codes, AdvantEdge software employs a damage model to integrate damage into the material model. The damage function D is stated in Eq. 5 [20].

$$
D=\sum_{i} \frac{\Delta \varepsilon_{i}^{p}}{\varepsilon_{f_{i}}^{p}}
$$

To have a precise cutting simulation, it is very important that proper friction should be modelled at the cutting tool-chip interface. In Thirdwave AdvantEdge friction at tool-chip interface is simulated using Coulomb law as represented in the Eq. 6 [20]. Coulomb law associates shear stresses by taking them proportional to the normal stresses and constant of proportionality is frictional coefficient $(\mu)$.

$$
\tau=\mu \sigma_{n}
$$

Selection of frictional constant is a difficult task because of complex sliding behavior at tool-chip interface. It is also reported that chip hardness is different and generally harder than the workpiece hardness, and hardness variation can lead to variation in friction coefficient [23,24]. Table 1 provides the information about the cutting parameters utilized for numerical simulations. In the current study the numerical simulations were limited to five different values of inclination angles ranging from $0-25^{\circ}$. The $0^{\circ}$ inclination angle leads to the orthogonal setup and acts like a reference for other values of inclination angles.

\section{Simulated RESUlts AND DisCUSSION}

The 3D simulated outcomes were studied for the different machining performance indicators such as cutting, thrust and oblique force components, cutting temperature and chip flow.

\section{A. Simulated Cutting Forces}

Cutting force components are very important machining performance indicators. These components have very significant role as they reveal the power consumption and vibrational effects in the machining process. They also play an important role towards the machine tool construction and fixture designing/ requirements. In this sub-section, simulated cutting forces at different inclination angles ranging from $0^{\circ}-$ $25^{\circ}$ were compared. The sample cutting force signals from the Thirdwave AdvantEdge softwares have been reported in Figures $3 a$ and $3 b$. 


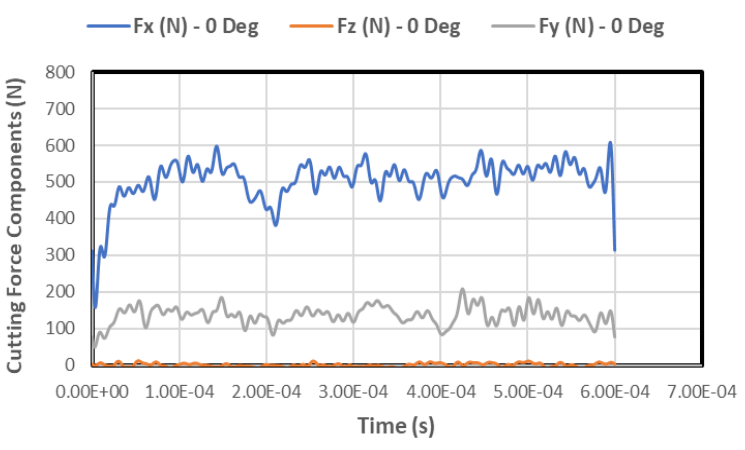

(a)

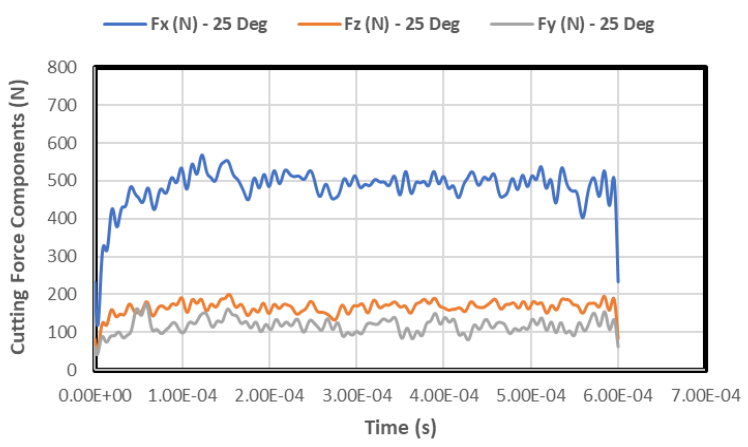

(b)

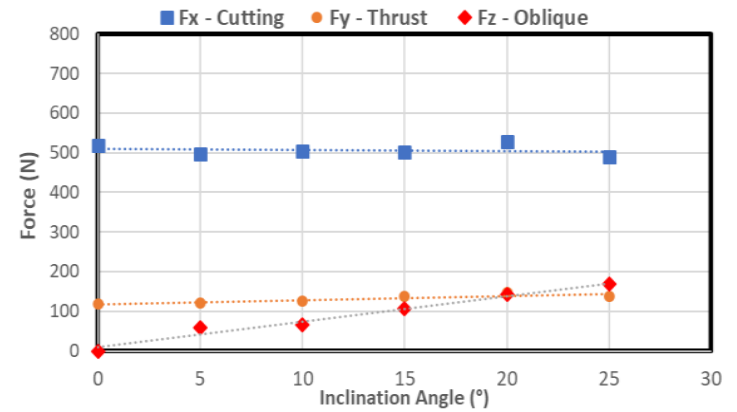

(c)

Figure 3. Simulated cutting force components (a) $0^{\circ}$ Inclination Angle, (b) $25^{\circ}$ Inclination Angle, (c) Simulated average cutting force components, $0^{\circ}-25^{\circ}$ inclination angles

The cutting force trends for all three cutting force components at all inclination angles have been reported in Figure $3 \mathrm{c}$. It can be observed that minor fluctuation has been reported for the cutting and thrust force components. The Fzoblique force component was majorly affected by the inclination angle.

\section{B. Simulated Cutting Temperature}

Cutting temperature has been attributed as one of the major machining performance indicators in the metal cutting sector. It is the deciding factor towards the cutting tool life, and many wear mechanisms such as diffusion and adhesion are linked with the cutting temperature. The sample cutting temperature signals from the Thirdwave AdvantEdge softwares have been reported in Figures $4 \mathrm{a}$ and $4 \mathrm{~b}$.
The average cutting temperature trends for all inclination angles has been reported in Figure 4c. The trend reveals that cutting temperature decreases slightly by increasing inclination angle.

\section{Simulated Chip Flow}

Simulated chip flow is another important machining parameter that has an important role in overall machining performance. Simulated results of chip flow for different inclination angles $0^{\circ}-25^{\circ}$ have been reported in Figures 5 and 6. Simulated chip flow observations revealed that increasing the inclination angles results in increasing the chip flow angle $\left(\prod_{\mathrm{c}}\right)$ as shown in Figure 6.

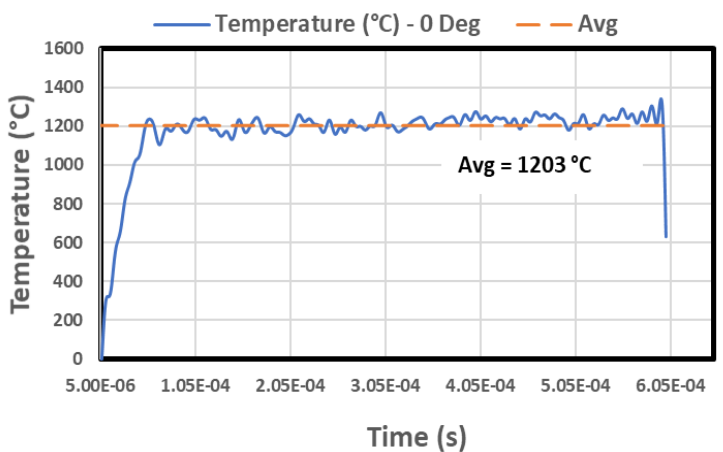

(a)

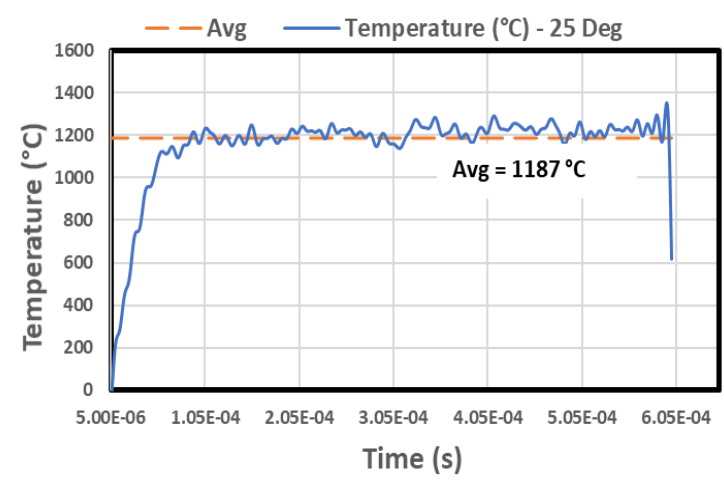

(b)

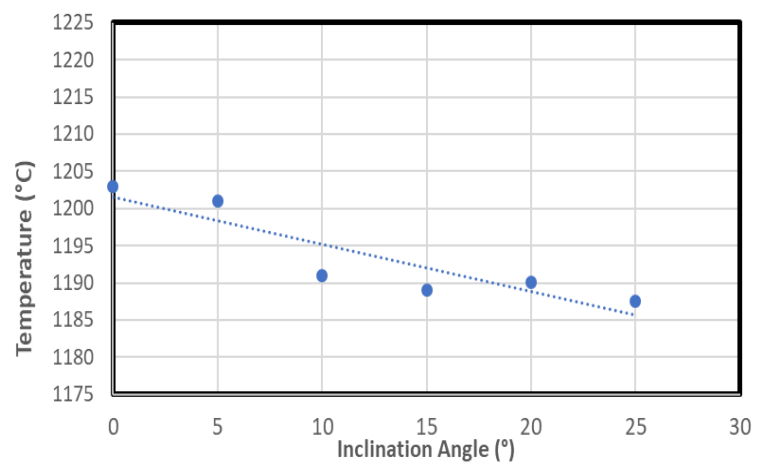

Figure 4. Simulated cutting temperatures (a) $0^{\circ}$ Inclination Angle, (b) $25^{\circ}$ Inclination Angle, (c) Simulated average cutting temperatures $0^{\circ}-25^{\circ}$ inclination angles 


\section{CONCLUSIONS}

The conclusions drawn from this study have been reported as under.

Inclination angle has negligible influence over the cutting force component which represents that it has negligible role towards power consumption. Thrust force component was found little sensitive to the inclination angle. Oblique force component was highly influenced by the inclination angle. The results were found in agreement with experimental and analytical studies present in the available literature. The cutting temperature slightly decreased by the introduction of inclination angles.

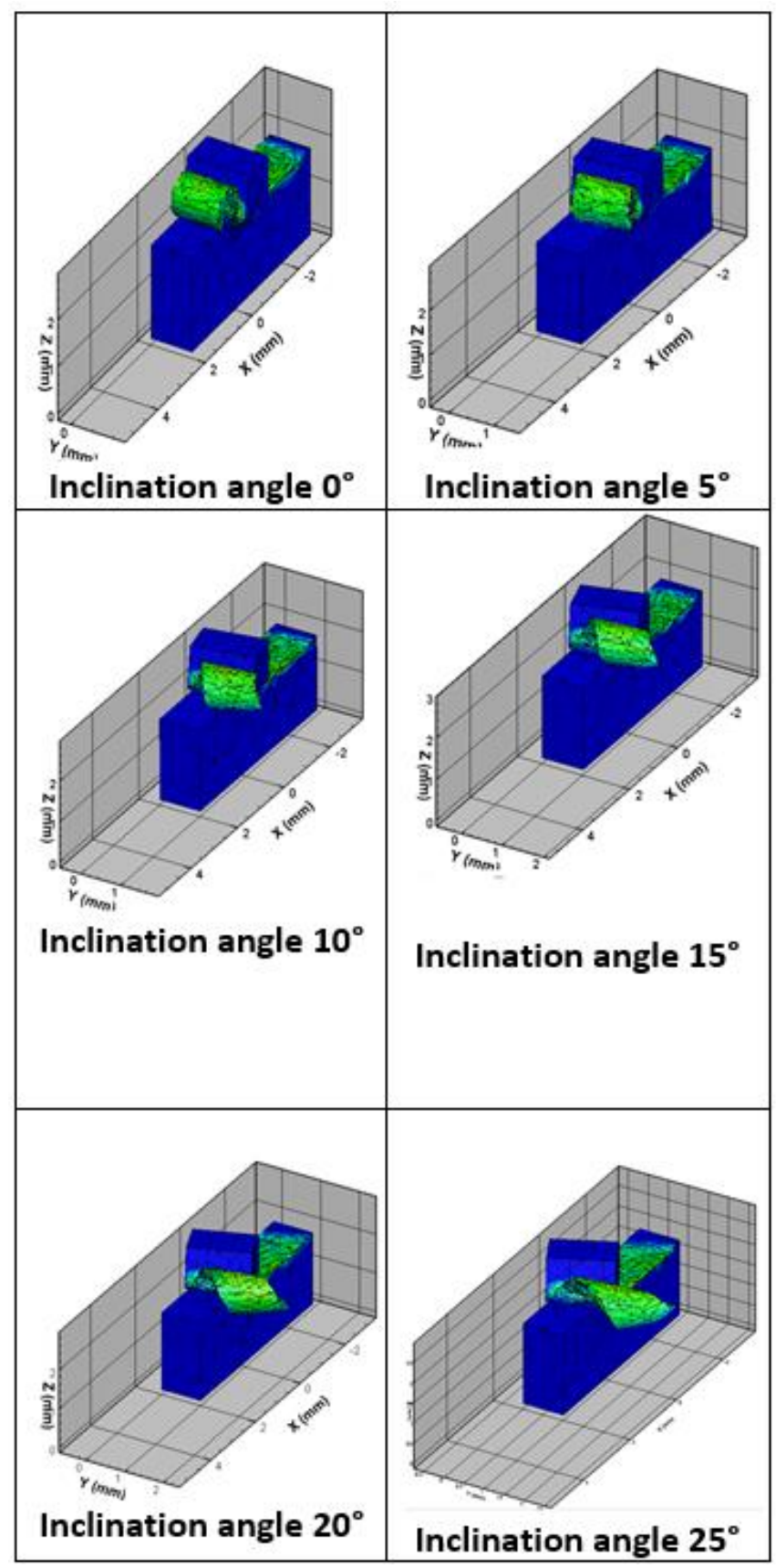

Figure 5. Simulations of chip flow, $0^{\circ}-25^{\circ}$ inclination angles

\section{ACKNOWLEDGEMENTS}

Authors sincerely acknowledge the financial support provided by Dubai Silicon Oasis Authority (DSOA) and Rochester Institute of Technology - Dubai (RIT-D).

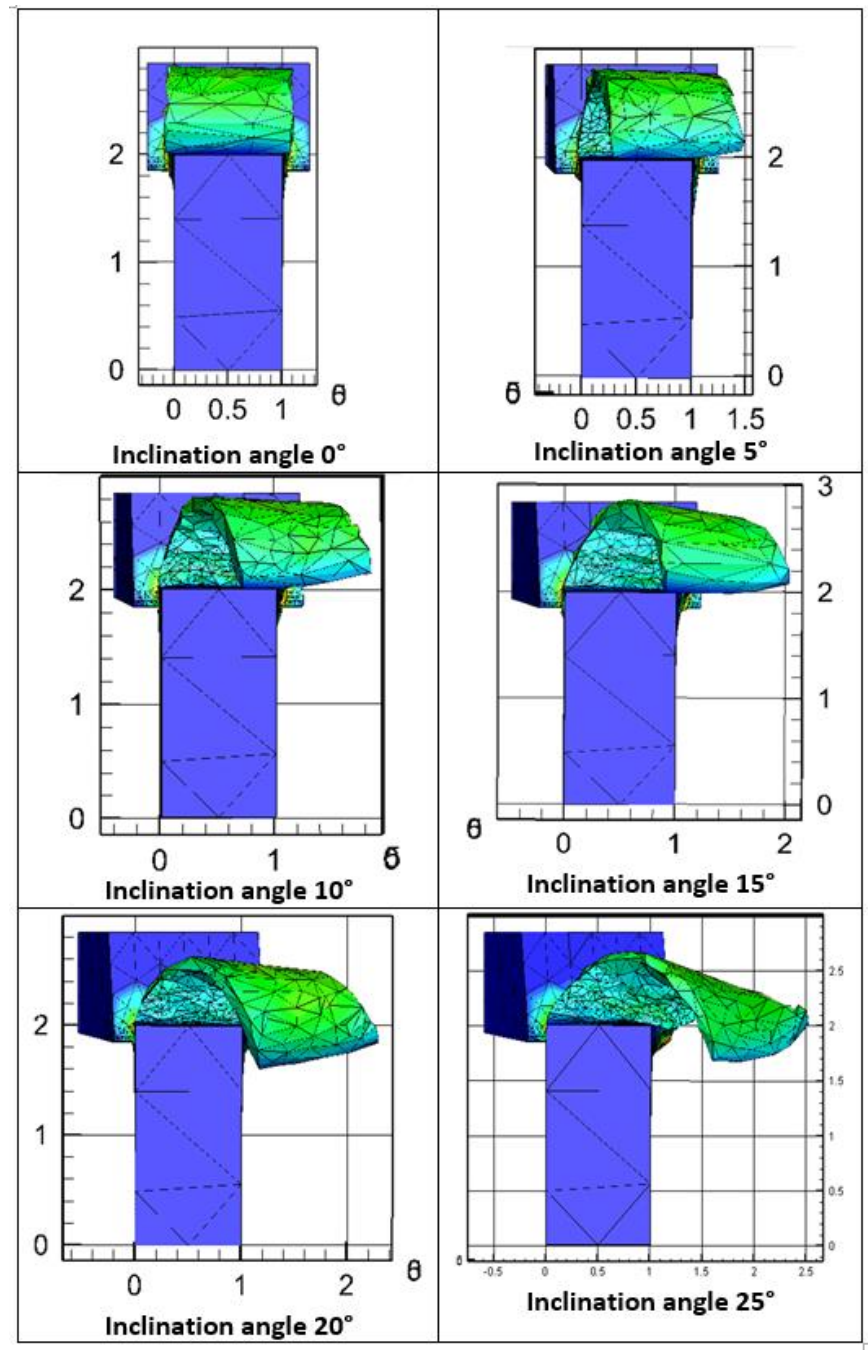

Figure 6. Front view of chip flow, $0^{\circ}-25^{\circ}$ inclination angles

\section{REFERENCES}

[1] M. C. Shaw, N. H. Cook, and P. A. Smith, "The mechanics of three dimensional cutting operations," Trans. ASME, vol. 74, pp. 1055- 1064, 1952.

[2] G. V. Stabler, "The fundamental geometry of cutting tools," Proc. Inst. Mech. Eng., vol. 165, pp. 14-21, 1951.

[3] G. V. Stabler, "The basic nomenclature of cutting tools," Inst. Prod. Eng. J., vol. 34, pp. 264-279, 1955.

[4] R. H. Brown and E. J. A. Armarego, "Oblique machining with a single cutting edge," Int. J. Mach. Tool Des. Res., vol. 4, pp. 9-25, 1964.

[5] J. K. Russell and R. H. Brown, "The measurement of chip flow direction,” Int. J. Mach. Tool Des. Res., vol. 6, pp. 129-138, 1966.

[6] W. K. Luk, "The direction of chip flow in oblique cutting," Int. J. Prod. Res., vol. 10, no. 1, pp. 67-76, 1972.

[7] G. C. Lin, P. Mathew, P. L. B. Oxley, and A. R. Watson, "Predicting Cutting Forces for Oblique Machining Conditions," Proc. Inst. Mech. Eng., vol. 196, pp. 141-148, 1982. 
[8] J. Arsecularatne, "Prediction of tool life in oblique machining with nose radius tools," Wear, vol. 198, no. 1-2, pp. 220-228, 1996.

[9] I. Lazoglu and C. Islam, "Modeling of 3D temperature fields for oblique machining," CIRP Ann. - Manuf. Technol., vol. 61, no. 1, pp. 127-130, 2012.

[10] R. Komanduri, M. Lee, and L. M. Raff, "The significance of normal rake in oblique machining," Int. J. Mach. Tools Manuf., vol. 44, no. 10, pp. 1115-1124, 2004.

[11] G. Fang and P. Zeng, "Three-dimensional thermo-elastic-plastic coupled FEM simulations for metal oblique cutting processes," J. Mater. Process. Technol., vol. 168, no. 1, pp. 42-48, 2005.

[12] G. Song, S. Sui, and L. Tang, "Precision prediction of cutting force in oblique cutting operation," Int. J. Adv. Manuf. Technol., vol. 81, no. 14, pp. 553-562, 2015.

[13] W. Grzesik and K. Zak, "Surface integrity generated by oblique machining of steel and iron parts," J. Mater. Process. Technol., vol. 212, no. 12, pp. 2586-2596, 2012.

[14] A. Moufki, A. Devillez, D. Dudzinski, and A. Molinari, "Thermomechanical modelling of oblique cutting and experimental validation,” Int. J. Mach. Tools Manuf., vol. 44, no. 9, pp. 971-989, 2004.

[15] L. Abdellaoui and W. Bouzid, "Thermomechanical approach for the modeling of oblique machining with a single cutting edge," Mach. Sci. Technol., vol. 20, no. 4, pp. 655-680, 2016.

[16] W. Grzesik and K. Zak, "Friction quantification in the oblique cutting with CBN chamfered tools," Wear, vol. 304, no. 1-2, pp. 36-42, 2013.

[17] I. Llanos, J. A. Villar, I. Urresti, and P. J. Arrazola, "Finite element modeling of oblique machining using an arbitrary lagrangian-eulerian formulation," Mach. Sci. Technol., vol. 13, no. 3, pp. 385-406, 2009.

[18] R. S. Pawade, H. A. Sonawane, and S. S. Joshi, "An analytical model to predict specific shear energy in high-speed turning of Inconel 718," Int. J. Mach. Tools Manuf., vol. 49, no. 12-13, pp. 979-990, 2009.

[19] M. C. Shaw, Metal Cutting Principles. Oxford University Press, Oxford, 1986.

[20] ThirdWaveSystems, Third Wave AdvantEdgeTM User's Manual Version 7.3. 2017.

[21] T.D., Marusich and O. M., "Modeling and Simulation of High Speed Machining,” Int. J. Numer. Methods Eng., vol. 38, no. 21, p. 3675 3694, 1995.

[22] ThirdWaveSystems, "Third Wave AdvantEdgeTM User's Manual Version 7.0," p. 378, 2015.

[23] K. W. Kim, W. Y. Lee, and H. Sin, "A finite element analysis for the characteristics of temperature and stress in micro-machining considering the size effect," Int. J. Mach. Tools Manuf., vol. 39, no. 9, pp. 15071524, 1999.

[24] A. Davoudinejad, P. Parenti, and M. Annoni, "3D finite element prediction of chip flow, burr formation, and cutting forces in micro endmilling of aluminum 6061-T6," Front. Mech. Eng., vol. 12, no. 2, pp. 203-214, 2017. 